\title{
Telling autonomous systems what to do
}

\author{
Peter Werkhoven \\ Utrecht University \& TNO \\ P.O. Box 96800 \\ The Netherlands \\ peter.werkhoven@tno.nl
}

\author{
Leon Kester \\ TNO \\ P.O.Box 96864 \\ The Netherlands \\ leon.kester@tno.nl
}

\author{
Mark Neerincx \\ Delft University of Technology \& TNO \\ P.O.Box 5031 \\ The Netherlands \\ mark.neerincx@tno.nl
}

\begin{abstract}
Recent progress in Artificial Intelligence, sensing and network technology, robotics, and (cloud) computing has enabled the development of intelligent autonomous machine systems. Telling such autonomous systems "what to do" in a responsible way, is a non-trivial task. For intelligent autonomous machines to function in human society and collaborate with humans, we see three challenges ahead affecting meaningful control of autonomous systems. First, autonomous machines are not yet capable of handling failures and unexpected situations. Providing procedures for all possible failures and situations is unfeasible because the state-action space would explode. Machines should therefore become self-aware (self-assessment, self-management) enabling them to handle unexpected situations when they arise. This is a challenge for the computer science community. Second, in order to keep (meaningful) control, humans come into a new role of providing intelligent autonomous machines with objectives or goal functions (including rules, norms, constraints and moral values), specifying the utility of every possible outcome of actions of autonomous machines. Third, in order to be able to collaborate with humans, autonomous systems will require an understanding of (us) humans (i.e., our social, cognitive, affective and physical behaviors) and the ability to engage in partnership interactions (such as explanations of task performances, and the establishment of joint goals and work agreements). These are new challenges for the cognitive ergonomics community.
\end{abstract}

Permission to make digital or hard copies of part or all of this work for personal or classroom use is granted without fee provided that copies are not made or distributed for profit or commercial advantage and that copies bear this notice and the full citation on the first page. Copyrights for third-party components of this work must be honored. For all other uses, contact the Owner/Author.

ECCE'18, September 5-7, 2018, Utrecht, Netherlands (C) 2018 Copyright is held by the owner/author(s). ACM ISBN 978-1-4503-6449-2/18/09. https://doi.org/10.1145/3232078.3232238

\section{CCS CONCEPTS}

- Human-centered computing $\rightarrow$ Interaction design $\rightarrow$ Interaction design theory, concepts and paradigms;

\section{KEYWORDS}

Cognitive ergonomics, control, autonomous systems;

ACM Reference format:

P. Werkhoven, M. Neerincx and L. Kester. 2018. Telling autonomous systems what to do. In European Conference on Cognitive Ergonomics 2018 Proceedings, 8 pages.

https://doi.org/10.1145/3232078.3232238

\section{INTRODUCTION}

\section{1 'Human in the loop' systems}

Cognitive ergonomics - as defined by the International Ergonomics Association - "is concerned with mental processes, such as perception, memory, reasoning, and motor response, as they affect interactions among humans and other elements of a system".

The tools and machinery that humans interact with have evolved from wooden and stone tools for hunting and food processing (some millions of years ago) to the present use of smart phones connecting and supporting humans at perceptual, cognitive and social levels. Essentially, we developed extensions of human beings, or human enhancement tools. Examples of such human enhancement tools at the perceptual level are binoculars, audiovisual sensors-display systems, and, recently, retinal and cortical implants [25]. For support of our motor behavior, we invented the lever and wheel, (motorized) machines and vehicles, and, recently, exoskeletons [10]. In order to enhance our cognitive abilities, we developed writing, the abacus for calculus, electronic 
computer and expert systems, e-coaches, (mobile) VR/AR environments $[5,36,42,47]$ and brain machine interfaces [19].

By giving machine systems the capability to sense the behavior and physiology of their human users and their environments, as well as sufficient intelligence to understand the users' goals and characteristics, machine systems became adaptive and personal. The development of adaptive cognitive systems (cognitive engineering) started in the eighties with including knowledge on human cognition in the iterative design of humancomputer interaction systems [20,33,35]. Later, interaction design methods were extended (with key concepts of 'technological design space' and domain-specific context) for cooperative systems [30] such as user interfaces for naval ships and space stations.

In all cases, users are 'in the loop', the systems are interactively controlled by the user's will, supporting the user's goals. Cognitive ergonomics has been concerned with designing systems that help people achieve their goals, not so much in defining their goals. Goals, from a Darwinian perspective, can be derived from the common goal of maximizing survival and reproductive success. Whilst defining their goals, many people find ethics or morality extremely helpful for considering varying combinations of values, principles and purposes. Ethics is the study of moral principles, or what is right and wrong behaviour, yielding 'codes of conduct' that are refined through history and inherited from long family, community or cultural traditions. It basically helps us answer the question "What is right to do?". Our behavior is further regulated by a system of rules called law (rights and duties), which promotes citizen compliance with the will of their country. In comparison to ethics, the written law is narrower in focus, and obeying the law may even require humans to act against their ethics. As long as humans are in control (in the loop) of their supporting machine systems, these systems can function within the ethical and legal constraints imposed by their users.

\section{2 'Human out of the loop' systems}

At this time, humans are on the verge of setting the next step, that is, the creation of intelligent machine systems that operate autonomously, placing humans in 'the outer loop', or even 'out of the loop'.

Machine systems have reached unprecedented levels of sensing, learning [40], reasoning, communication, and motor abilities. They started taking decisions without humans being in the loop. Cochlear and visual implants and automatic language interpreters filter information that they think we need. Intelligent medical implants can show learning behavior after they have been certified. Intelligent operator support systems for ship control have become semi-autonomous [9]. Automated vehicles [12,24] and military systems [23] make more and more decisions independently, and robots interact with humans autonomously [26].

Automated systems can be characterized by their ability to observe their environment, cognitive ability to analyze their situation and decide on what action to take, and their physical ability to execute actions (called the Observe-Orient-Decide-Act, or OODA-loop) [8]. Equipped with algorithms for situation assessment (observe, orient) and situation management (decide, act), autonomous systems continuously adapt to their environment to reach their goals. However, automated systems are not capable yet of handling failures, unreliable resources, unexpected situations. Additionally, the development of more complex algorithms to instruct automated systems 'what to do' is increasingly laborious (the state-action space dimension is increasingly higher). Learning abilities make automated systems more flexible yet slower in complex environments that are unpredictable and difficult to understand. Nevertheless, they still cannot handle failures and new situations. Humans are still needed in a 'supervising-' or 'collaborative'-type role as a fail-safety agent (human intervenes when system fails) and moral agent (human decides when system acts immorally).

The development of intelligent autonomous machine systems raises the question of how to meaningfully control these systems, that is, how to guarantee behavioral compliance with human ethics and law.

\section{MEANINGFUL CONTROL OF AUTONOMOUS SYSTEMS}

\subsection{Normative versus consequential approach}

In the 'Statement on Artificial Intelligence, Robotics and Autonomous Systems' of the European Group on Ethics in Science and New Technologies [42], a set of basic ethical principles and democratic prerequisites have been proposed for the design of autonomous systems. For example, autonomous systems should not violate 'human dignity', impair the freedom of human beings ('autonomy'), or interfere with the human right to be free from technologies that influence personal development or the right to be free from surveillance ('data protection and privacy'). Further, research and development of autonomous systems should be guided by concern for ethics and social accountability ('responsibility') and guarantee 'security, safety and mental integrity' of humans. There should be equal access to autonomous technologies and fair distribution of benefits ("justice, equity and solidarity') and protection against risks stemming from autonomous systems ('rule of law and accountability'). Key decisions on the regulation of AI development should be the result of a democratic debate ('democracy'). Lastly, autonomous systems should be designed to guarantee physical and emotional safety for humans and internal robustness against hacking ('security, safety, bodily and mental integrity', and technology development must be in line with 'sustainability' goals.

The 'autonomy' principle addresses human control over and predictability of autonomous systems, but does not state how to do that. This predicament was already commented on by Aristotle in $322 \mathrm{BC}$ : "For if every instrument could accomplish its own work, obeying or anticipating the will of others ... chief workmen would not want servants, nor masters slaves" [2]. However, how 
to let autonomous systems obey or anticipate the 'will' of humans? Assuming that humans know why they want something, they could tell systems what they want and how to do it. Instructing machine systems 'what to do', however, becomes impossible for systems that have to operate in complex, unstructured and unpredictable environments for the so-called state-action space would be too high-dimensional and explode in complex, unstructured and unpredictable environments. Humans telling systems 'what we want', touches on the question of how well humans know what they want, that is, do humans know what's best for them in the short and longer term? Can we fully understand the potential beneficial and harmful effects of actions and measures taken, and their interactions and trade-offs, on the individual and on society? Can we eliminate the well-known biases in human cognition inherent to the neural system that humans developed as hunter-gatherers (superstition, framing, conformation and availability biases) and learned through evolutionary survival in small groups (authority bias, prosocial behavior, loss aversion)? What sort of future do we want [41]?

Let's assume that humans are capable of defining adequate goals. How do we make sure that autonomous systems achieve these goals within the boundaries set by law and ethics? This is not even apparent for humans themselves. Firstly, the law system contains rules that require reasoning about trade-offs when trying to comply with those rules. For example, we've seen much discussion about the tension between the right of free expression and anti-discrimination laws. Secondly, morality is not univocal given the wide differences in opinion about gun control, immigration policy, etc. Differences in perception of right or wrong are, amongst others, rooted in differences in short- versus long-term goal setting, individual versus collective benefits, religious and cultural back grounds, etc. According to Wittgenstein in 1929 [49] the 'absolute good' may not even exist and be 'relative' to the goals pursued. Walking into a street may be good if one wants to find a butcher, not if one wants to find a shoe shop. Eating meat from the butcher may be good if one wants to stay healthy, not if one wants to optimize the well-being of animals. Staying healthy may be good if one wants to live long, but not if one wants to prevent overpopulation and scarcity of food. So, actions are right or wrong only relative to goals.

So, how do we let systems function within a framework of law and ethics? One approach would be to define a normative framework (deontological ethics, the study of obligation and duty) for systems to judge the morality of their actions based on universal, national and religious laws and rules, or on cultural values. This approach may not be feasible, however, because of the state-action space explosion, and because machine systems still have to interpret the laws and rules, and make tradeoffs and solve dilemmas.

A more feasible approach would be not to define a normative framework for actions, but to value the possible consequences of our actions (consequential ethics). That is, humans should give autonomous systems goal functions (e.g. reaching a target) that quantify utility for each possible outcome (e.g. collateral damage). Autonomous systems then optimize on utility, assuming that we can find adequate dimensional units to express and accurately weigh utility. An interesting aspect of a consequential ethics approach is that making explicit the values that have to be optimized (e.g. 'happiness' or 'life satisfaction') may yield actions that conflict with deontological ethics.

As an example, the law forbids murder of a human which can be interpreted as deontological ethics. However, it could be the case that the victim was a terrorist caught in the act of committing an attack on citizens. A judge, following consequential reasoning and appealing to higher values, will probably not punish the defendant. It is this appeal to higher values that somehow should be implemented in autonomous systems. Either humans specify the normative behavior for all possible circumstances - yielding a computational explosion of state-action for complex environments - or humans specify goals in the form of goal functions expressing the utility for each of the possible outcomes, such that autonomous systems can maximize on utility.

\subsection{Goal functions that specify utility}

The challenge we face with fully autonomous systems is to design systems that can operate in complex situations and achieve human defined goals, such that safety and cost-effectivity are guaranteed,

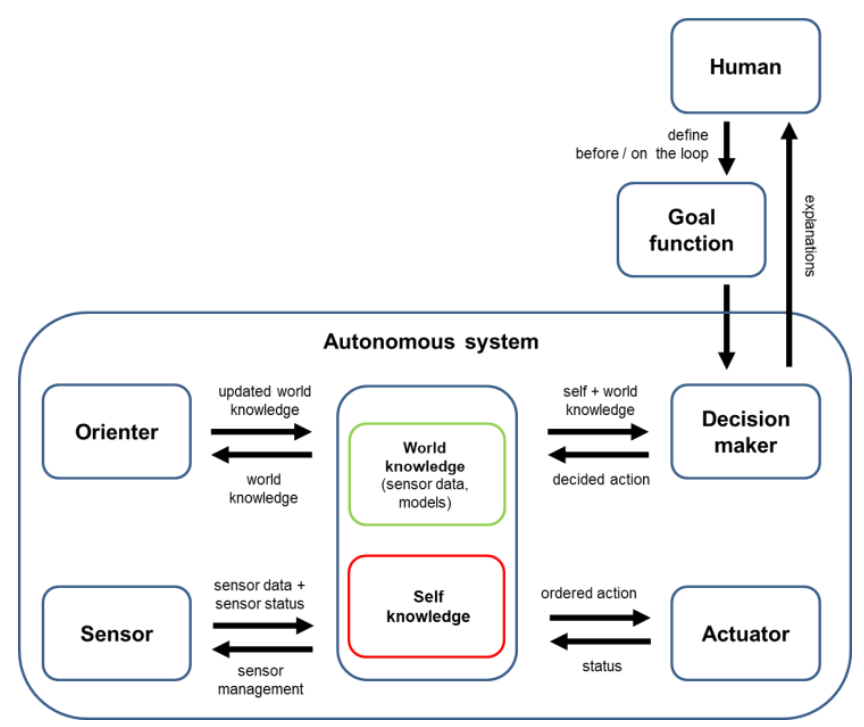

Figure 1: Meaningful control of intelligent systems. Boxes illustrate the OODA elements of an autonomous system, that is, the sensor, orienter, decision maker and actuator elements. The decision maker is capable of self-assessment and selfmanagement (based on self-knowledge). Humans are in meaningful control by defining the goal function for the system, expressing the utility for each possible outcome. If connectivity, time and complexity allows, goal functions can be adjusted by the human based on explanations of the system's actions (human on the loop), else (human out of the loop) the goal function is specified before the loop. 
that the system can explain itself, and that it operates within legal and ethical boundaries. For this, autonomous systems should be capable of self-assessment (the utility of its resources, sensors, actuators and cognitive capabilities) and of self-management (how do I manage myself such that I increase the utility and reach my goals in a more effective way). Together, this can be called 'selfawareness' (see Fig. 1).

For autonomous systems to be compliant with human goals and intentions, predictability must be guaranteed (system behavior stays within human expectations), and even so directability (mission, intent and preferences can be given by humans) and accountability (comprehensible record of reasoning and actions taken).

The role of the human will turn into 'meaningful controller', that is, defining the (ethical) goals of the system in the form of goal functions, 'before the loop' (see Fig. 1). The formulation of good and lawful behavior will go through a paradigm from a framework of rules, norms and constraints to a framework of goal functions that quantify utility. Systems will be more capable themselves of deriving the rules, norms and constraints from the human defined goal functions.

A goal function should be defined in a consistent, explicit (including ethics) and complete way (expressing the utility of every possible outcome). The goal function specifies the utility (contribution to the human defined goal) of all possible world states being the result of all possible actions of the autonomous system. World state is described by a finite set of $n$ parameters, each of which can be influenced by a possible action. These world state parameters are input to the n-dimensional goal function specifying the total utility of that world state. The system uses the goal function to evaluate the utility of all possible actions, and chooses the action with the highest expected utility. The evaluation process of the expected utility of possible actions is carried out by the decision maker of the system (see Fig. 1) based on world knowledge (world models, sensor data and orienter information) and self-assessment. The chosen action is passed on to the actuator of the system.

In a simple example, the goal of an autonomous car is to transport a person from A to B. The utility could be based on three world state parameters that are speed-dependent: arrival time, the environmental pollution due to carbondioxide emission, and the health effect of an accident caused by the car. The system uses this three-dimensional goal function to evaluate total expected utility as a function of these (speed dependent) parameters, and chooses the speed yielding the maximum expected utility. In the case of combinatorial optimization of utility, the system should be able to map the dimensions of the different parameters onto a single utility dimension, usually a monetary unit or a scale for wellbeing. For utility parameters that are defined at a societal level (not limited to the individual passenger), utility should neither be defined by the passenger, nor by the producer or owner of the car, but by (a representation) of society as a whole.

In practice it can be difficult to determine a complete multidimensional goal function. A practical approach to determining the goal function in the domain of cooperative driving was found by Van Willigen et al [48], who developed a method for a driver-definable goal function. As an example, the parameters travel time, and comfort were selected. The autonomous systems calculated the possible solutions in terms of travel time and comfort (expressed in the number of lane changes) and the driver could then select the most utile point on the curve. This method can easily be extended to include other parameters such as cost and safety. Additionally, parameters should be added that guarantee the (ethical) behavior of vehicles towards other road users. However, these should not be defined by the vehicle designer, owner or driver, but by the authorities.

The theory of guidance control [38] argues that humans can be in meaningful control of autonomous systems when two conditions are satisfied: tracking (the system should be able to respond to both the relevant moral reasons of the humans designing and deploying the system), and tracing (the system should be designed in such a way as to grant the possibility to always trace back the outcome of its operations to at least one human along the chain of design and operation). These tracking and tracing conditions, however, raise the question if and how individual humans can convey the relevant moral reasons to autonomous systems, and how to let systems reason about the utility of possible outcomes of their operations. Furthermore, it is disputable if the individual humans designing and deploying the system can adequately represent society in defining what are the right moral reasons for the autonomously operating system, considering the role of legislative and judicial power of the government.

We propose an important role for the legislative power (definitions of goal functions before the loop) and judicial power (judging system explanations after the loop). Helping users and authorities to define adequate goal functions for intelligent autonomous systems in complex environments could be a new role for cognitive engineers. We are facing the development of objective and systematic methods for our society to think about goal (and subgoal) functions that quantify utility, such that societal and industrial stakeholders can recognize the utility framework created and can help developing it.

\subsection{Interaction with and understanding of humans}

Autonomous machine behaviors will also appear and evolve in human work and living environments where they interact with humans. Progress in Artificial Intelligence, sensing and network technology, and cloud computing has led to the development of machine intelligence (ePartners) enabling machine systems to behave and communicate as team members or partners of humans [39].

\subsubsection{Meaningful partnership interactions.}

As a team-member, ePartners need to acquire and demonstrate understanding of the meaning, weighting and implementation method of the joint goals they are committed to. This type of understanding and commitment is being built and communicated via three partnership interactions (see Fig. 2). 
First, the setting, progress monitoring, refinement and adjustment of the joint goals encompasses a shared understanding, awareness and communication of the goals, their relevance, the corresponding utilities and their states. Stakeholders' values, strategic intentions and momentary context conditions determine which goals are of relevance and feasible to achieve. The choices of the goals can require re-assessment and adjustment to deal with changing circumstances, possibly just in advance or even during the work processes. Goal ontology design patterns $[13,34]$ and utility elicitation methods [15] are being developed to support the setting of joint goals. Adaptivity is an important capability of human-machine teams, which may involve creativity to find a new work process (possibly with another goal) when the current process is expected to fail. Humans may think differently than machines and, as a result of diversity, the perspective taking and solution space for off-nominal (wicked) problems can be larger when machines and humans share their perceptions and cognitions. For example, a goal and work model has been developed and instantiated in ePartners for future space missions to Mars which have to deal with potentially non-nominal situations, such as a malfunctioning rover [7]. Due to the Mars rover's malfunctioning, the original mission goal of an extravehicular activity may not be attained (e.g., setting up a specific cache at a remote location). There will be several options to deal with this situation, such as to cancel the mission and return to the habitat, or (partially) repair the rover and set up a cache at a closer location (which services another goal). The ePartner supports the exploration of alternative mission goals from which the team can select one. Evaluations with the Eurobot rover and with a virtual reality environment showed that the model-based ePartner support can enhance the mission team's resilience substantially [7].

Second, human-machine partnerships involve univocal adjustable work agreements on how the work should be divided and performed to establish explicit commitment to the chosen way of working [16,28]. An example of work agreement modeling is the development of human-robot partnerships for disaster response, in which the response team needs to dynamically adapt the task allocation and coordination to the momentary context [14]. An ontology has been developed that defines the knowledge required for a team member to set, reason about and adjust work agreements. A disaster response field test showed that the firebrigade officers could specify work agreements as required, which, subsequently, brought forward the desired adaptive team behavior of the concerning robot [28].

Third, partners have to provide explanations of their behavior to establish the right level of trust (i.e., to invoke trust calibration that mitigates over- or under-reliance) and to support learning. Currently, a perceptual-cognitive explanation ( $\mathrm{PeCoX}$ ) framework is being developed that defines the perceptual and cognitive grounding of ePartners' behavior (Fig. 2) [32], and constructs explanation dialogues that are meaningful for the human [27]. The core of this framework is generic over domains and the perceptual layer is generic over different machine learning models. Currently, PeCoX is being developed and tested in the domains of transport, health-care and defense. The perceptual level provides an Intuitive Confidence Measure [45] and identifies the "foil" that can be used in a contrastive explanation [46]. Such an explanation fits with human explanation dialogues, centering on the question "Why this output (the fact) instead of that output (the foil)?" It reduces the factors in the explanation to the ones that are of interest to the human, so that it can be better interpreted by the human. The cognitive level provides the beliefs, goals and emotions for explanations [17,22]. Initial evaluations showed that humans can understand the explanations that $\mathrm{PeCoX}$ will provide, but further personalization and contextualization is needed.

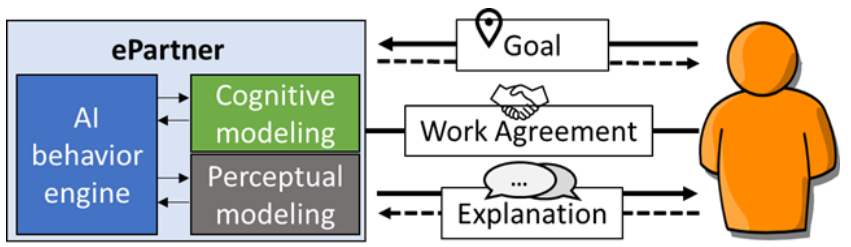

Figure 2: Human-autonomy partnerships are established with joint goals to pursue, work agreements to shape the situated behaviors, and explanations for trust calibration and learning [32].

\subsubsection{Evolution of partnership interactions}

The development of intelligent machines is seldom a one-step process, but more an evolutionary process. Partnership interactions can help to guide this evolutionary process in a desired way (e.g., for adequate trust calibration). It should be noted that the actual (joint) goal can relate to the state, condition and behavior of the human. An example is the use of intelligent machines in healthcare (e.g., for the prevention and management of lifestyle-related diseases). The proposed partnership interactions can help improve human commitment to the goals and work agreements (i.e. to persuade humans to adhere to behaviors that promote their health).

In general, ePartners, as described above, are being developed to advance the effectiveness, efficiency and resilience of work processes in a responsible way, for example, for the prevention and management of lifestyle related diseases [31], robot-assisted disaster management [28], train traffic control [18] and manned space missions [7]. In these domains, generic computational models of the human's social, cognitive, emotional and physical processes are being developed, so that the ePartner can be aware of and reason about these processes. Following coactive design [21] and the situated Cognitive Engineering methods [30] "understanding-the-human" capability is being developed and tested incrementally, aiming at a library of ontology design patterns [13] that provide re-usable building blocks of ePartners' capability to understand the human. 


\section{DISCUSSION}

\subsection{Telling systems what to do or what to achieve?}

\subsubsection{Will human control limit AI potential?}

In an open letter signed by (amongst many others) Max Tegmark, Elon Musk and Stephen Hawking it was stated that "We recommend expanded research aimed at ensuring that increasingly capable AI systems are robust and beneficial: our AI systems must do what we want them to do." (https://futureoflife.org/ai-openletter/). In this paper, we suggest, that 'what we want them to do' should be framed in goal functions (quantifying the utility of possible outcomes), and not dictate systems how to achieve those goals. The reason being that, through goal functions and selfawareness, intelligent autonomous systems may be able to understand how to achieve these goals better (with more intelligence and creativity) than humans could ever think of. For situations where machine systems and humans benefit from meaningful human-machine interactions we can implement work agreements (see 2.3).

\subsubsection{Will explainability limit AI potential?}

We mentioned the requirement of accountability of autonomous systems, the ability to provide a comprehensible record of reasoning and actions taken. Not surprisingly, the European General Data Protection Regulation (GDPR) and other regulations increasingly demand transparency and the ability of systems to explain themselves. However, demanding 'explainability' may cause artificial intelligent systems to limit their reasoning and resulting choices and actions, thereby limiting their potential benefit for human society, to what can be understood by humans. Should we not wish to allow our colleagues, our children and even so AI-systems to be more intelligent and creative than we are, solving societal issues that we can't solve ourselves? At some point, humans may want to trust that goals can be achieved against less cost and better outcomes when we accept solutions even if they are less understandable by humans. And with the goal function-based approach, humans can explicitly verify how well the system is doing with respect to the goal function specified.

\subsection{AI assisted goal specification}

\subsubsection{Debiasing human cognition}

As mentioned earlier, the effectivity and consistency of human goal and utility specification may be complicated by well known biases in human cognition inherent to the neural system that humans developed as hunter-gatherers (superstition, framing, conformation and availability biases) and developed though evolutionary survival in small groups (authority bias, prosocial behavior, loss aversion)?

For humans to effectively and ethically define goals and utility of AI-systems, they need to become better 'ethical regulators' [3]. Ashby describes nine requisites for a regulator to be effective and ethical, regardless of whether the regulators are humans, organisations, machines, or hybrids. Amongst others, requisites are truth about past and present, unambiguously prioritized goals and rules, predictability of effects of actions, intelligence to choose best actions, and transparency of ethical behavior.

Aliman and Kester [1] discuss types of 'human enhancement' to ameliorate the utility specification abilities of humans, including debiasing training [29], methods from behavioral ethics [11] and future types of AI-assisted debiasing methods. In the end, artificial general intelligence may generate methods to debiase and enhance human cognition and the ability to unbiased (objective) definition of goals and utility in a bidirectional learning framework.

When developing AI-systems for our assistance, we must be careful not to 'transfer' human biases to AI-systems themselves. First, models that are used by the AI-system are constructed by humans and therefore may include human cognitive biases. Secondly, the AI-system may be trained with large but not representative data sets. The AI-system may learn, for example, how to recognize swans from pictures with only white swans in the training set, so it will probably not recognize black swans as swans. Thirdly, if a system learns from humans, e.g. how to behave, it will have the same biases as the particular humans it has learned from. These biases are sometimes mistakenly considered inherent to AI-system biases. Lastly, some cognitive biases are inherent to a neural system and therefore artificial neural networks exhibit the same type of biases.

\subsubsection{Empowering human goal setting: policy by simulation}

Defining goals and utility of possible outcomes may not be as easy as we may wish, given the extreme complex representation and behavior of the environments in which the systems should operate and lack of explicit human knowledge about cause-effect relationships and interactions in those environments. For this reason, human expert knowledge could benefit from AI-systems that combine model-driven and data-driven approaches in enabling 'what-if' scenario generation, finding causal relationships, and predicting the outcome of applied measures and policies to achieve certain goals. This is sometimes referred to as 'policy by simulation'. In such manner, AI-systems could support humans in balancing cost and benefits and defining their goals effectively. It could lead to more objective goal related ethical decision making.

One example of 'policy by simulation' in the urban planning domain is the urban strategy tool developed by TNO and Arcadis [6]. The tool provides intelligent assistance for human decision makers, based on integrated and validated models on air pollution, traffic flow and noise, safety risks, energy consumption and heat production. Another example of 'policy by simulation' in the military strategy domain is developed by TNO [4].

\section{CONCLUSIONS}

For intelligent autonomous machines to function in human society and collaborate with humans, we see three challenges ahead. First, machines should become self-aware (self-assessment, selfmanagement) enabling them to deal with unexpected situations as 
they arise. This is a challenge for the computer science community. Second, in order to maintain (meaningful) control, humans come into a new role of providing intelligent autonomous machines with objectives or goal functions (including rules, norms, constraints and moral values), specifying the utility of every possible outcome of actions of the autonomous machines. Third, in order to be able to collaborate with humans, autonomous systems need to understand the human (i.e., their social, cognitive, affective and physical behaviors) and to engage in partnership interactions (such as the setting of joint goals and work agreements, and explanations of task performances). We see the last two as important new challenges for the cognitive ergonomics community.

Finally, artificial intelligence could play a role in assisting humans by modeling and understanding the complex and often interrelated physical and behavioral mechanisms of the world we live in, by simulating the consequences of ethical choices, and by helping us define the goal functions that optimize human wellbeing.

\section{REFERENCES}

[1] N.M. Aliman and L. Kester. 2018. Hybrid Strategies Towards Safe ISelfAware" Superintelligent Systems. AGI 18.

[2] Aristotle and Jonathan Barnes. 1984. The complete works of Aristotle: The revised Oxford translation. Princeton, N.J: Princeton University Press.

[3] M. Ashby. 2017. Ethical Regulators and Super-Ethical Systems. In: Proceedings of the 61st Annual Meeting of the ISSS-2017 Vienna, Austria.

[4] A.I. Barros, A.C. Van den Broek, J.A. Van Dalen, B. Vecht, and J. Wevers. 2014. Producing near-real-time intelligence: predicting the world of tomorrow. TMC Asser Press.

[5] Mark Billinghurst, Adrian Clark, and Gun Lee. 2015. A Survey of Augmented Reality. Found. Trends Hum.-Comput. Interact 8, 2-3, 73-272.

[6] Jeroen Borst. J. (2017). \{ HYPERLINK "http://conferences.iaia.org/2017/uploads/presentations/IAIA17\%20\%20Urban\%20Strategy\%20Tool\%20v7.pdf" ?

[7] T. Bosse, L. Breebaart, J. Van Diggelen, M.A. Neerincx, J. Rosa, and N.J. Smets. 2017. Developing ePartners for human-robot teams in space based on ontologies and formal abstraction hierarchies. International Journal of AgentOriented Software Engineering, 5(4), 366-398.

[8] John R. Boyd. 1987. A discourse on winning and losing. Maxwell Air Force Base, AL: Air University Library Document No. M-U 43947 (Briefing slides)

[9] J van Diggelen, H van den Broek, JM Schraagen, and J van der Waa. 2017. An Intelligent Operator Support System for Dynamic Positioning. Int. Conference on Applied Human Factors and Ergonomics, 48-59.

[10] M.P. De Looze, T. Bosch, F. Krause,m K.S. Stadler, and L.W. O'Sullivan. 2016. Exoskeletons for industrial application and their potential effects on physical work load. Ergonomics, 59(5), p 671-81.

[11] M. Drumwright, R. Prentice, and C. Biasucci. 2015. Behavioral ethics and teaching ethical decision making. Decision Sciences Journal of Innovative Education 13(3), 431-458.

[12] C. Englund, L. Chen, and J. Ploeg. 2016. The Grand Cooperative Driving Challenge 2016: boosting the introduction of cooperative automated vehicles. IEEE Wireless Communications , 23(4), 146-152.

[13] A. Gangemi and V. Presutti. 2009. Ontology design patterns. In Handbook on ontologies, 221-243. Springer, Berlin, Heidelberg.

[14] T.R. Giele, T. Mioch, M.A.Neerincx, \& J.J.C. Meyer. 2015. Dynamic Task Allocation for Human-Robot Teams. In ICAART (1), 117-124.

[15] J. González-Ortega, V. Radovic, and D.R. Insua. 2018. Utility elicitation. In Elicitation, 241-264. Springer, Cham.

[16] R.S. Gutzwiller, S.H. Espinosa, C. Kenny, and D.S. Lange. 2017. A Design Pattern for Working Agreements in Human-Autonomy Teaming. In International Conference on Applied Human Factors and Ergonomics, 12-24. Springer, Cham.

[17] M. Harbers, K. van den Bosch, and J.J. Meyer. 2010. Design and evaluation of explainable BDI agents. In Web Intelligence and Intelligent Agent Technology (WI-IAT), 2010 IEEE/WIC/ACM International Conference on Web Intelligence and Intelligent Agent Technology, Toronto, ON, 2010, 125-132.
[18] M. Harbers and M.A. Neerincx. 2017. Value sensitive design of a virtual assistant for workload harmonization in teams. Cognition, Technology \& Work, 19(2-3), 329-343.

[19] S.V. Hiremath, W. Chen, W. Wang, S. Foldes, Y. Yang, E.C. Tyler-Kabara, J.L. Collinger, and M.L. Boninger. 2015. Brain computer interface learning for systems based on electrocorticography and intracortical microelectrode arrays. Frontiers in Integrative Neuroscience 9.

[20] E. Hollnagel and D.D. Woods. 1983. "Cognitive systems engineering: New wine in new bottles." International Journal of Man-Machine Studies, 18 , 583-600.

[21] M. Johnson, J.M. Bradshaw, P.J. Feltovich, C.M. Jonker, M.B. Van Riemsdijk, and M. Sierhuis. 2014. Coactive design: Designing support for interdependence in joint activity. Journal of Human-Robot Interaction, 3 (1)

[22] F. Kaptein, J. Broekens, K. Hindriks, and M.A. Neerincx. 2017. Personalised self-explanation by robots: The role of goals versus beliefs in robot-action explanation for children and adults. 26th IEEE International Symposium on Robot and Human Interactive Communication (RO-MAN), 676-682.

[23] L. Kester and M. Ditzel. 2014. Maximising effectiveness of distributed mobile observation systems in dynamic situations. 17th International Conference on Information Fusion (FUSION), 1-8.

[24] M. Kyriakidis, J.C.F. de Winter, N. Stanton, T. Bellet, B. van Arem, K.A. Brookhuis, and R. Happee. 2017. A human factors perspective on automated driving. Ergonomics, -. DOI: 10.1080/1463922X.2017.1293187

[25] Philip M. Lewis, Helen M. Ackland, Arthur J. Lowery, and Jeffrey V. Rosenfeld. 2015. Restoration of vision in blind individuals using bionic devices: A review with a focus on cortical visual prostheses. Brain Research, Vol. 1595, p 51-73,

[26] M. Ligthart, K. Hindriks, and M.A. Neerincx. (2018). Reducing Stress by Bonding with a Social Robot: Towards Autonomous Long-Term ChildRobot Interaction. Companion of the 2018 ACM/IEEE International Conference on Human-Robot Interaction. p 305-306.

[27] T. Miller. 2017. Explanation in artificial intelligence: Insights from the social sciences. arXiv preprint arXiv: 1706.07269

[28] T. Mioch, M.M.M. Peeters, and M.A. Neerincx. 2018. Improving Adaptive Human-Robot Cooperation through Work Agreements. RO-MAN2018 27th IEEE International Symposium on Robot and Human Interactive Communication.

[29] C.K. Morewedge, H. Yoon, I. Scopelliti, C.W. Symborski, J.H. Korris, K.S. Kassam. 2015. Debiasing decisions: Improved decision making with a single training intervention. Policy Insights from the Behavioral and Brain Sciences 2(1), 129-140.

[30] Mark A. Neerincx and Jasper Lindenberg. 2008. Situated cognitive engineering for complex task environments. In: Schraagen, J.M.C., Militello, L. Ormerod, T. Lipshitz, R. (eds.) Naturalistic Decision Making and Macrocognition, 373-390. Ashgate, Aldershot.

[31] M.A. Neerincx, F. Kaptein, M.A. Van Bekkum, H. Krieger, B. Kiefer, R. Peters, and M. Sapelli. 2016. Ontologies for social, cognitive and affective agent-based support of child's diabetes self-management. In Proc. ECAI 16, 35-38.

[32] M.A. Neerincx, J. Van der Waa, F. Kaptein, and J. Van Diggelen. 2018. Using perceptual and cognitive explanations for enhanced human-agent team performance. EPCE 2018, LNAI 10906, p 1-11, Springer.

[33] Donald A. Norman. 1986. Cognitive engineering. In D.A. Norman, and S.W Draper (eds), User-Centered System Design: New Perspectives on HumanComputer Interaction. Hillsdale, NJ: Lawrence Erlbaum Associates, 31-62.

[34] R. Peters, J. Broekens, and M.A. Neerincx. 2017. Guidelines for Tree-based Collaborative Goal Setting. In Proceedings of the 22nd International Conference on Intelligent User Interfaces, 401-405.

[35] J. Rasmussen. 1986. Information Processing and Human-Machine Interaction: An Approach to Cognitive Engineering. Amsterdam: Elsevier.

[36] N. Rosa, W. Hürst, R. Veltkamp, and P. Werkhoven. 2017. Player-Avatar Link, Interdisciplinary Embodiment Perspectives. Encyclopedia of Computer Graphics and Games.

[37] E. Saad, K.V. Hindriks, and M.A. Neerincx. 2018. Ontology Design for Task Allocation and Management in Urban Search and Rescue Missions. In Proceedings of the 10th International Conference on Agents and Artificial Intelligence - Volume 2: ICAART, ISBN 978-989-758-275-2, pages 622629. DOI: $10.5220 / 0006661106220629$.

[38] Filippo Santoni de Sio and Jeroen van den Hoven. 2018. Meaningful Human Control over Autonomous Systems: A Philosophical Account. Frontiers in Robotics and AI, 5.

[39] R.J. Shively, J. Lachter, S.L. Brandt, M. Matessa, V. Battiste, and W.W. Johnson. 2017. Why Human-Autonomy Teaming? In International Conference on Applied Human Factors and Ergonomics, 3-11, Springer, Cham.

[40] D. Silver, A. Huang, C. Maddison, and D. Hassabis. 2016. Mastering the game of Go with deep neural networks and tree search. Nature 529 (7587), 
484-489.

[41] M. Tegmark. 2017. Life 3.0, Being Human in the Age of Artificial Intelligence. New York : Alfred A. Knopf.

[42] The European Group on Ethics in Science and New Technologies. 2018. Statement on Artificial Intelligence, Robotics and 'Autonomous' Systems.

[43] M.L. Tielman, M.A. Neerincx, R. Bidarra, B. Kybartas, and W.P. Brinkman.

2017. A therapy system for post-traumatic stress disorder using a virtual agent and virtual storytelling to reconstruct traumatic memories. Journal of medical systems, 41(8), 125.

[44] J. Van Diggelen, H. van den Broek, J.M. Schraagen, and J. Waa. 2017. An Intelligent Operator Support System for Dynamic Positioning. Advances in Intelligent Systems and Computing, p 48-59. 10.1007/978-3-319-60204-2_6.

[45] J. Van der Waa, J. Van Diggelen, and M.A. Neerincx. 2018. The design and validation of an intuitive certainty measure. Proceedings of IUI 2018

Workshop on Explainable Smart Systems.

[46] J. Van der Waa, M.J. Robeer, J. Van Diggelen, M.J.S. Brinkhuis, and M.A. Neerincx. 2018. Contrastive Explanations with Local Foil Trees. IJCAI 2018 Workshop on Human Interpretability in Machine Learning (WHI).

[47] P. Werkhoven, T. Philippi, and J.B.F. van Erp. 2014. Navigation in virtual mazes: The benefits of audiovisual landmarks. Displays, 35(3): 110-117.

[48] Willem van Willigen, Evert Haasdijk, and Leon Kester. 2013. A MultiObjective Approach to Evolving Platooning Strategies in Intelligent Transportation Systems. Proceedings of the 2013 Genetic and Evolutionary Computation Conference, p1397-1404.

[49] Ludwig Wittgenstein. 1929. A Lecture on Ethics. In Philosophical Review (Jan. 1965), 3-12. 